\title{
Parameter und Trends der Stutbuchaufnahme des Mecklenburger Warmblut Pferdes
}

\author{
Herrn Professor Dr. Ernst Ritter zum 75. Geburtstag gewidmet
}

\begin{abstract}
Title of the paper: Parameters and trends of mare inspections of Mecklenburger Warmblut Horse

Genetic and phenotypic parameters and genetic trends were estimated for traits of mare inspections of Mecklenburger Warmblut by means of REML. The data were recorded at mare inspections from 1991 to 2002 and included evolution data of 3654 mares. Total pedigree size was 9073 animals. The number of observation per level of the factors place by date of evaluation was very variable and often sparse. Therefore, this factor was treated as random. Age and year of affiliation into the herdbook were treated as fixed effects in the BLUP model. Estimated heritabilities ranged from low $(0.08)$ to medium $(0.48)$ and estimates of genetic and phenotypic correlations were generally positive, some of them above 0.8 . The pattern of correlation was very similar to literature values from the Hannoveraner breed with exception of the traits saddle position and rear limbs. The genetic trends were significantly positive for traits horse points, quality of conformation and total score. Genetic differences were significant between warm blood breeds and age classes. Influences of factors year, month, age and place were significant in the majority of traits.
\end{abstract}

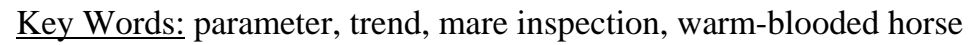

\section{Zusammenfassung}

Für Merkmale der Stutbuchaufnahme des Mecklenburger Warmbluts wurden mittels REML genetische und phänotypische Parameter und genetische Trends geschätzt. Das Datenmaterial stammt von Stutbuchaufnahmen der Jahre 1991 bis 2002, in denen 3654 Stuten vorgestellt wurden. Das Pedigree umfasst insgesamt 9073 Tiere. Die Stufen Ort und Datum der Beurteilung waren sehr heterogen und schwach besetzt. Ihr Einfluss wurde neben den fixen Faktoren Alter und Jahr als Zufallsfaktor im Modell berücksichtigt. Die geschätzten Heritabilitätskoeffizienten lagen mit 0,08 bis 0,48 im unteren bis mittleren Bereich und mit Ausnahme der Merkmale Sattellage und Hintergliedmaßen waren sie denen der Hannoveraner sehr ähnlich. Die genetischen und phänotypischen Korrelationen waren generell positiv, teilweise sehr hoch und unterschieden sich von den entsprechenden Schätzwerten der Hannoveraner ebenfalls geringfügig. Die genetischen Trends der Merkmale Reitpferdepoints, Qualität des Körperbaus und Gesamtnote waren signifikant positiv. Zwischen den Warmblutrassen und den Altersklassen ließen sich genetische Unterschiede sichern. Einflüsse der Faktoren Jahr und Monat der Beurteilung, Alter und Ort erwiesen sich in der Mehrzahl der Merkmale als signifikant.

Schlüsselwörter: Parameter, Trend, Stutbuchaufnahme, Warmblutpferd

1. Einleitung

Die Beurteilung der äußeren Erscheinung (Exterieur) einschließlich der Veranlagungen in der freien Bewegung liefert insbesondere bei jungen Pferden erste Informationen zum Gebrauchswert eines Reitpferdes. Man geht davon aus, dass ein guter Körperbau und korrekte, elastische Bewegungen Grundvoraussetzungen für hohe, ausdau- 
ernde Leistungsfähigkeit darstellen. In mehreren Untersuchungen konnte allerdings gezeigt werden, dass zwischen diesen Merkmalen und der Reiteignung (Dressur und Springen) nur geringe Korrelationen bestehen. Jedoch haben die Ergebnisse der Beurteilung bei der Vermarktung junger Pferde und bei Pferden für den Freizeitsport unmittelbare ökonomische Bedeutung für den Besitzer, da die Ästhetik des Pferdes, auch mangels Leistungsinformation, wertbestimmend wird. Nach Untersuchungen von BRUNS (1988) lassen sich mehr als $10 \%$ der Auktionserlöse auf das Exterieur und die Bewegung des Pferdes zurückführen. Auch SCHWARK et al. (1988) fanden mit 0,48 eine deutlich positive Korrelation zwischen Exterieurnote und Verkaufspreis.

In der Zucht des Mecklenburger Warmblutes spielen der Typ, das Fundament und die Bewegung der Pferde, die bei der Stutbuchaufnahme bewertet werden, eine wesentliche Rolle. Seit Beginn der Neunziger werden Zuchtwerte geschätzt und im Zuchtziel ist diesen Eigenschaften ein Stellenwert von 40 \% eingeräumt.

Ziel dieser Arbeit ist es, das Mecklenburger Warmblut anhand von Populationsparametern bei Merkmalen der Stutbuchaufnahme zu analysieren und züchterische Trends zu ermitteln.

\section{Material und Methode}

$2.1 \quad$ Das Datenmaterial

Die Ergebnisse der Stutbuchaufnahme von 3654 Stuten, so wie sie zur verbandsinternen Zuchtwertschätzung des Verbandes der Pferdezüchter Mecklenburg-Vorpommerns e. V. im 2. Halbjahr 2002 vorlagen, bilden die Datengrundlage der Populationsanalysen. Die Eintragungen erstrecken sich über den Zeitraum von 1991 bis 2002 und gehen in den Geburtsjahren bis zum Jahre 1979 zurück. Beurteilt wurden vorwiegend Dreijährige (2780), aber auch Stuten im Alter von 4 - 5 Jahren (580) und älter (294). Der Ort (164) und der Zeitpunkt der Stutbuchaufnahme sind sehr variabel. Die Stuten gehören der Rassegruppe Warmblut an und stammen aus 10 Zuchtverbänden. Das Pedigree beinhaltet insgesamt 9073 Pferde.

Zur Stutbuchaufnahme wird die Stute zunächst auf einer harten Fläche der Aufnahmekommission vorgestellt, die für die Exterieurmerkmale Rasse und Geschlechtstyp, Korrektheit des Ganges, sowie Kopf, Hals, Sattellage, Rahmen, Vordergliedmaßen und Hintergliedmaßen ganzzahlige Noten im Bereich von 1 bis 10 vergibt. Der Mittelwert der Noten Kopf, Hals, Sattellage, Rahmen, Vordergliedmaßen und Hintergliedmaßen bildet das Merkmal Qualität des Körperbaus. Danach erfolgt die Beurteilung der Bewegung. Bis 1995 wurden die Stuten in einer Dreiecksbahn auf der rechten Hand im Schritt und Trabe geführt, seit 1996 beurteilt man die Tiere in einer Reithalle in freier Bewegung. Die Kommission bewertet die Merkmale Gesamteindruck, Schwung und Elastizität in der Bewegung (Trab und Galopp) und Schritt. Eine Gesamtnote ergibt sich als Mittelwert der Noten Qualität des Körperbaus, Rasse und Geschlechtstyp, Gesamteindruck, Korrektheit des Ganges, Elastizität und Schritt. Für eine kompaktere Nutzung der Ergebnisse aus den Stutbucheintragungen werden zwei weitere zusammengefasste Noten, Reitpferdepoints und Fundament, gebildet. Die Note Reitpferdepoints ist der Mittelwert aus der Note Rasse- und Geschlechtstyp und der Durchschnittsnote der Merkmale Kopf, Hals, Sattellage und Rahmen. Die Fundamentnote errechnet sich als Mittelwert aus der Note Korrektheit des Ganges und der 
Durchschnittsnote der Vorder- und Hintergliedmaßen. Neben der Vergabe der Noten wird auch die Widerristhöhe gemessen.

Die Tabelle 1 enthält eine Übersicht der Merkmale.

Tabelle 1

Stichprobengrößen, Mittelwerte, Standardabweichungen, Variationskoeffizienten und Extremwerte bei Merkmalen der Stutbuchaufnahme (Sample sizes, means, standard deviations, coefficients of variation and extreme values by traits of mare inspections)

\begin{tabular}{lcccccc}
\hline Merkmal & Anzahl & Mittelwert & $\begin{array}{c}\text { Standard- } \\
\text { abweichung }\end{array}$ & $\begin{array}{c}\text { Variations- } \\
\text { koeffizient }\end{array}$ & Minimum & Maximum \\
\hline Rasse und Geschlechtstyp & 3653 & 7,49 & 0,67 & $9 \%$ & 4 & 10 \\
Kopf & 3654 & 7,38 & 0,75 & $10 \%$ & 5 & 10 \\
Hals & 3654 & 7,46 & 0,66 & $9 \%$ & 5 & 10 \\
Sattellage & 3654 & 7,20 & 0,57 & $8 \%$ & 5 & 9 \\
Rahmen & 3654 & 7,10 & 0,69 & $10 \%$ & 4 & 10 \\
Vordergliedmaßen & 3654 & 6,71 & 0,62 & $9 \%$ & 5 & 9 \\
Hintergliedmaßen & 3653 & 6,27 & 0,57 & $9 \%$ & 4 & 8 \\
Qualität des Körperbaus & 3654 & 7,06 & 0,41 & $6 \%$ & 5 & 9 \\
Korrektheit des Ganges & 3653 & 6,69 & 0,61 & $9 \%$ & 4 & 10 \\
Elastizität & 3654 & 7,04 & 0,71 & $10 \%$ & 5 & 10 \\
Schritt & 3654 & 6,95 & 0,69 & $10 \%$ & 4 & 10 \\
Gesamteindruck & 3653 & 7,20 & 0,63 & $9 \%$ & 5 & 9 \\
Gesamtnote & 3654 & 7,07 & 0,41 & $6 \%$ & 5 & 9,5 \\
Reitpferdepoints & 3653 & 7,39 & 0,53 & $7 \%$ & 5,4 & 9,9 \\
Fundament & 3652 & 6,59 & 0,45 & $7 \%$ & 5 & 9 \\
Größe & 3646 & $164,4 \mathrm{~cm}$ & $3,6 \mathrm{~cm}$ & $2 \%$ & $134 \mathrm{~cm}$ & $179 \mathrm{~cm}$ \\
\hline
\end{tabular}

\section{$2.2 \quad$ Statistische Auswertung}

Die Schätzung von Varianzkomponenten wurde unter der Annahme normalverteilter Merkmale in multivariaten Analysen nach REML mit dem Programmpaket VCE 4.2 GROENEVELD (1998) durchgeführt. Die Parameter der Merkmale Qualität des Körperbaus, Reitpferdepoints, Fundament und Gesamtnote, die lineare Funktionen originaler Noten darstellen, wurden aus den Schätzwerten der entsprechenden Noten nach den Regeln zur Berechnung von Varianzen und Kovarianzen linearer Funktionen von Zufallsvariablen berechnet. Folgendes Modell, bezogen auf ein einzelnes Merkmal, wurde verwandt:

$$
\mathrm{y}=\mathrm{X} \beta+\mathrm{Zo}+\mathrm{Tu}+\varepsilon
$$

Hierbei sind:

$$
\begin{array}{rll}
\mathrm{Y}- & \text { Vektor der Beobachtungswerte } \\
\beta- & \text { Vektor der fixen Effekte } \\
\mathrm{O}- & \text { Vektor der zufälligen Effekte „Ort x Datum“ } \\
\mathrm{u}- & \text { Vektor der zufälligen additiv genetischen Effekte } \\
\varepsilon- & \text { Vektor der zufälligen Resteffekte } \\
\mathrm{X}, \mathrm{Z}, \mathrm{T}- & \text { Versuchsplanmatrizen }
\end{array}
$$

Das Modell enthält als fixe Faktoren das Jahr der Stutbuchaufnahme und die Altersklasse (bis 3 Jahre; 4 + 5 Jahre; über 5 Jahre), zu der die Stute gehört. Innerhalb des Aufnahmejahres wurde die Variable „Ort mal Datum der Stutbuchaufnahme“ gebildet und wegen der sehr schwach besetzten Stufen als Zufallsfaktor aufgenommen, was 
durch die eher zufällige Zuordnung von Stute zu Ort und Zeitpunkt innerhalb Jahr gerechtfertigt erscheint. Alle Effekte des Modells wurden mit dem Programm PEST von GROENEVELD und KOVAC (1990) geschätzt und die Summe aller Kontraste dieser Effekte mit dem in PEST integrierten $\chi^{2}$-Test getestet. Einflüsse auf die zufälligen Faktoren, Zuchtwert und „Ort mal Datum“, wurden, stratifiziert nach Rassen, Altersklassen, Orte und Monate, mittels der einfachen F-Statistik statistisch geprüft, wobei die Signifikanzgrenzen über Bootstrap mit 20000 „resamplings“ bestimmt wurden. In gleicher Weise wurden die Signifikanzgrenzen linearer Trends ermittelt.

\section{3. $\quad$ Ergebnisse}

Die Stichprobengrößen sowie Mittelwerte, Standardabweichungen, Variationskoeffizienten Minima und Maxima der untersuchten Noten sind in der Tabelle 1 angegeben. Zwischen den Mittelwerten, die von 6,27 für Hintergliedmaßen bis 7,49 für Rasse und Geschlechtstyp reichen, bestehen deutliche Unterschiede. Die Standardabweichungen liegen unterhalb von 0,80 und sind bei den zusammengefassten Noten, Qualität des Körperbaus und Gesamtnote mit 0,41 am niedrigsten. Das führt erwartungsgemäß bei diesen Merkmalen zu den geringsten Variationskoeffizienten von jeweils nur $6 \%$. Die Minima und Maxima zeigen, dass Noten nur von 4 bis 10 vergeben wurden, im Merkmal Hintergliedmaßen nur von Note 4 bis Note 8 . Zu mehr als $80 \%$ sind Wertnoten zwischen 7,00 und 8,00, in den Merkmalen des Fundaments zwischen 6,00 und 7,00 , vergeben worden. Die Größe, als objektiv erhobenes Merkmal, dient lediglich der Einhaltung rassespezifischer Größenvorgaben. Daraus sind die geringe Standardabweichung von 3,6 cm und der Variationskoeffizient von $2 \%$ erklärbar.

\section{Tabelle 2}

Anteile der Varianzkomponenten „additiv genetisch“ $\left(\mathrm{h}^{2}\right)$, „Ort mal Datum der Stutbuchaufnahme“ $\left(\mathrm{o}^{2}\right)$ und „Rest“ $\left(\mathrm{e}^{2}\right)$ an der Gesamtvarianz. (Proportions of the variance components "additive genetic" $\left(\mathrm{h}^{2}\right)$, "place by date of mare inspection" $\left(\mathrm{o}^{2}\right)$ and "residual" $\left(\mathrm{e}^{2}\right)$ on the total variance)

\begin{tabular}{lccc}
\hline Merkmal & $\mathrm{h}^{2}$ & $\mathrm{O}^{2}$ & $\mathrm{e}^{2}$ \\
\hline Rasse und Geschlechtstyp & $0,29 \pm 0,02$ & $0,09 \pm 0,01$ & $0,62 \pm 0,01$ \\
Kopf & $0,41 \pm 0,02$ & $0,04 \pm 0,01$ & $0,55 \pm 0,02$ \\
Hals & $0,29 \pm 0,02$ & $0,08 \pm 0,01$ & $0,63 \pm 0,02$ \\
Sattellage & $0,11 \pm 0,01$ & $0,12 \pm 0,01$ & $0,77 \pm 0,01$ \\
Rahmen & $0,14 \pm 0,01$ & $0,08 \pm 0,01$ & $0,78 \pm 0,01$ \\
Vordergliedmaßen & $0,15 \pm 0,01$ & $0,13 \pm 0,01$ & $0,72 \pm 0,01$ \\
Hintergliedmaßen & $0,08 \pm 0,01$ & $0,18 \pm 0,01$ & $0,74 \pm 0,01$ \\
Qualität des Körperbaus & $0,28 \pm 0,01$ & $0,20 \pm 0,02$ & $0,52 \pm 0,02$ \\
Korrektheit des Ganges & $0,12 \pm 0,01$ & $0,15 \pm 0,01$ & $0,73 \pm 0,01$ \\
Elastizität & $0,20 \pm 0,01$ & $0,11 \pm 0,01$ & $0,69 \pm 0,01$ \\
Schritt & $0,16 \pm 0,01$ & $0,09 \pm 0,01$ & $0,75 \pm 0,01$ \\
Gesamteindruck & $0,19(0,01$ & $0,14(0,02$ & $0,67(0,01$ \\
Gesamtnote & $0,30(0,01$ & $0,22(0,02$ & $0,48(0,02$ \\
Reitpferdepoints & & & $0,55(0,03$ \\
Fundament & $0,33(0,02$ & $0,12(0,01$ & $0,62(0,03$ \\
Größe & $0,16(0,02$ & $0,22(0,02$ & $0,48 \pm 0,02$ \\
\hline
\end{tabular}

Der Einfluss der im Modell berücksichtigten festen Faktoren (Jahr der Stutbuchaufnahme und Altersklasse) erwies sich bei allen Noten als zum Niveau 5 \% signifikant. 
Die Einflüsse auf die Größe waren nicht signifikant. Für den Faktor Jahr der Stutbuchaufnahme zeigte sich an den zusammengefassten Noten, dass in den Jahren von 1987 bis 1989 die Stuten höher bewertet wurden. Dabei verlaufen die Veränderungen über die Jahre parallel sowohl zwischen Reitpferdepoints und Gesamtnote als auch zwischen Fundament und Qualität des Körperbaus. Die Note Qualität des Körperbaus verringert sich kontinuierlich von der 1 . Altersklasse (bis 3 Jahre) bis zur 3. Altersklasse (über 5 Jahre) um eine halbe Standardabweichung. Die Gesamtnote der ersten Altersklasse ist um etwa 0,15 Punkte höher als die der beiden anderen Klassen. Die Tabelle 2 zeigt die Aufteilung der geschätzten Varianz in ihre Komponenten „additiv genetisch“ $\left(h^{2}\right)$, „Ort mal Datum der Stutbuchaufnahme“ $\left(\mathrm{o}^{2}\right)$ und „Rest“ $\left(\mathrm{e}^{2}\right)$. Die angegebenen Standardabweichungen der Schätzfehler überschreiten in keinem der Fälle den Wert 0,02. Die Werte der Heritabilitätskoeffizienten liegen mit 0,08 bis 0,48 im unteren bis mittleren Bereich. Die $\mathrm{o}^{2}$-Werte sind meist geringer als ihre entsprechenden $\mathrm{h}^{2}$ Werte. Auffällig ist, dass die zusammengefassten Noten die höchsten Anteile dieser Varianzkomponente aufweisen.

Tabelle 3

Genetische (oberhalb Diagonale) und phänotypische Korrelationen (unterhalb Diagonale) bei Noten des Körperbaus (Genetic (above diagonal) and phenotypic (below diagonal) correlation coefficients for body conformation scores)

\begin{tabular}{|c|c|c|c|c|c|c|c|c|c|c|}
\hline & Kopf & Hals & $\begin{array}{l}\text { Sattel- } \\
\text { lage }\end{array}$ & $\begin{array}{l}\text { Rah- } \\
\text { men }\end{array}$ & $\begin{array}{l}\text { Vorder } \\
\text { glied- } \\
\text { maßen }\end{array}$ & $\begin{array}{l}\text { Hinter- } \\
\text { glied- } \\
\text { maßen }\end{array}$ & $\begin{array}{l}\text { Quali- } \\
\text { tät des } \\
\text { Körper } \\
\text { baus }\end{array}$ & $\begin{array}{l}\text { Reit- } \\
\text { pferde- } \\
\text { points }\end{array}$ & $\begin{array}{c}\text { Funda- } \\
\text { ment }\end{array}$ & $\begin{array}{l}\text { Gesamt- } \\
\text { note }\end{array}$ \\
\hline Kopf & - & 0,42 & 0,37 & 0,47 & 0,11 & 0,39 & 0,72 & 0,78 & 0,35 & 0,60 \\
\hline Hals & 0,35 & - & 0,59 & 0,71 & 0,47 & 0,45 & 0,82 & 0,82 & 0,57 & 0,74 \\
\hline Sattellage & 0,21 & 0,33 & - & 0,54 & 0,37 & 0,21 & 0,67 & 0,69 & 0,43 & 0,64 \\
\hline Rahmen & 0,27 & 0,31 & 0,38 & - & 0,62 & 0,59 & 0,86 & 0,79 & 0,60 & 0,67 \\
\hline Vordergliedmaßen & 0,13 & 0,15 & 0,18 & 0,25 & - & 0,86 & 0,66 & 0,49 & 0,86 & 0,59 \\
\hline Hintergliedmaßen & 0,20 & 0,18 & 0,18 & 0,25 & 0,34 & - & 0,72 & 0,57 & 0,83 & 0,61 \\
\hline $\begin{array}{l}\text { Qualität d. } \\
\text { Körperb. }\end{array}$ & 0,63 & 0,64 & 0,60 & 0,68 & 0,55 & 0,57 & - & 0,96 & 0,75 & 0,86 \\
\hline Reitpferdepoints & 0,64 & 0,63 & 0,54 & 0,67 & 0,29 & 0,32 & 0,82 & - & 0,63 & 0,91 \\
\hline Fundament & 0,25 & 0,22 & 0,23 & 0,32 & 0,64 & 0,63 & 0,61 & 0,39 & - & 0,73 \\
\hline Gesamtnote & 0,48 & 0,49 & 0,45 & 0,57 & 0,40 & 0,43 & 0,77 & 0,80 & 0,65 & - \\
\hline
\end{tabular}

Die genetischen und phänotypischen Korrelationskoeffizienten zwischen den Merkmalen des Körperbaus (Tab. 3) sind gering, jedoch wie aus ihrer Bildung zu erwarten, zu den zusammengefassten Noten hoch. Auch innerhalb der Merkmale des Typs und der Bewegungen sind sehr hohe genetische Korrelationen, mit Ausnahme zwischen Rasse- und Geschlechtstyp und dem Gesamteindruck (Tab. 4) von 0,94 sowie zwischen beiden zu den Reitpferdepoints und zur Gesamtnote, nicht festzustellen. Innerhalb der Merkmale der Bewegung bestehen nur geringe bis mittlere genetische $\mathrm{Zu}$ sammenhänge. Sowohl die Note für Rasse- und Geschlechtstyp als auch die Note für den Gesamteindruck sind genetisch eng mit der Note für den Rahmen korreliert (Tab. 5). Ebenfalls findet man engere genetische Korrelationen zwischen Kopf und Hals zu Rasse- und Geschlechtstyp. Dagegen sind die genetischen Korrelationen zwischen den Noten der Bewegung und den Noten für die Gliedmaßen eher moderat (Elastizität) bzw. sehr gering (Schritt). Die Standardabweichungen der Schätzfehler, in den Tabellen nicht aufgeführt, erreichen in keinem der Fälle Werte von 0,06 und höher. 
Tabelle 4

Genetische (oberhalb Diagonale) und phänotypische Korrelationen (unterhalb Diagonale) bei Noten des Typs und der Bewegung (Genetic (above diagonal) and phenotypic (below diagonal) correlation coefficients for type and movement scores)

\begin{tabular}{|c|c|c|c|c|c|c|c|c|c|}
\hline & $\begin{array}{c}\text { Rasse \& } \\
\text { Geschl- } \\
\text { echts- } \\
\text { typ }\end{array}$ & $\begin{array}{c}\text { Ge- } \\
\text { samt- } \\
\text { ein- } \\
\text { druck }\end{array}$ & $\begin{array}{l}\text { Kor- } \\
\text { rektheit } \\
\text { des } \\
\text { Ganges }\end{array}$ & $\begin{array}{c}\text { Elasti- } \\
\text { zität }\end{array}$ & Schritt & $\begin{array}{c}\text { Qualität } \\
\text { des } \\
\text { Körper- } \\
\text { baus }\end{array}$ & $\begin{array}{c}\text { Reit- } \\
\text { pferde- } \\
\text { points }\end{array}$ & $\begin{array}{c}\text { Funda- } \\
\text { ment }\end{array}$ & $\begin{array}{c}\text { Gesamt- } \\
\text { note }\end{array}$ \\
\hline Rasse\&Geschlechtstyp & - & 0,94 & 0,58 & 0,72 & 0,52 & 0,93 & 0,99 & 0,64 & 0,95 \\
\hline Gesamteindruck & 0,59 & - & 0,54 & 0,76 & 0,47 & 0,87 & 0,90 & 0,67 & 0,93 \\
\hline Korrektheit des Ganges & 0,25 & 0,28 & - & 0,58 & 0,19 & 0,64 & 0,58 & 0,90 & 0,68 \\
\hline Elastizität & 0,41 & 0,47 & 0,25 & - & 0,67 & 0,55 & 0,64 & 0,60 & 0,88 \\
\hline Schritt & 0,23 & 0,28 & 0,16 & 0,29 & - & 0,35 & 0,46 & 0,22 & 0,67 \\
\hline Qualität d. Körperb. & 0,70 & 0,64 & 0,36 & 0,39 & 0,28 & - & 0,96 & 0,75 & 0,86 \\
\hline Reitpferdepoints & 0,95 & 0,65 & 0,28 & 0,42 & 0,25 & 0,85 & - & 0,63 & 0,91 \\
\hline Fundament & 0,36 & 0,40 & 0,86 & 0,30 & 0,22 & 0,61 & 0,39 & - & 0,73 \\
\hline Gesamtnote & 0,76 & 0,78 & 0,55 & 0,70 & 0,57 & 0,77 & 0,80 & 0,65 & - \\
\hline
\end{tabular}

Tabelle 5

Genetische und phänotypische Korrelationen zwischen Noten des Körperbaus und Noten des Typs und der Bewegung (Genetic and phenotypic correlation coefficients between body conformation scores and type and movement scores)

\begin{tabular}{lcccccc}
\hline & Kopf & Hals & Sattellage & Rahmen & Vordergliedmaßen & Hintergliedmaßen \\
\hline \multirow{7}{*}{ Rasse\&Geschlechtstyp } & 0,75 & 0,79 & 0,66 & 0,74 & 0,51 & 0,58 \\
Gesamteindruck & 0,55 & 0,71 & 0,66 & 0,80 & 0,64 & 0,60 \\
Korrektheit des Ganges & 0,39 & 0,53 & 0,45 & 0,46 & 0,57 & 0,54 \\
Elastizität & 0,30 & 0,56 & 0,41 & 0,36 & 0,47 & 0,46 \\
Schritt & 0,31 & 0,29 & 0,37 & 0,18 & 0,16 & 0,23 \\
& \multicolumn{7}{c}{ Phänotypische Korrelationskoeffizienten } \\
Rasse\&Geschlechtstyp & 0,52 & 0,49 & 0,40 & 0,56 & 0,27 & 0,30 \\
Gesamteindruck & 0,36 & 0,40 & 0,38 & 0,56 & 0,32 & 0,32 \\
Korrektheit des Ganges & 0,21 & 0,17 & 0,16 & 0,22 & 0,27 & 0,28 \\
Elastizität & 0,24 & 0,29 & 0,24 & 0,26 & 0,19 & 0,21 \\
Schritt & 0,14 & 0,15 & 0,19 & 0,20 & 0,15 & 0,19 \\
\hline
\end{tabular}

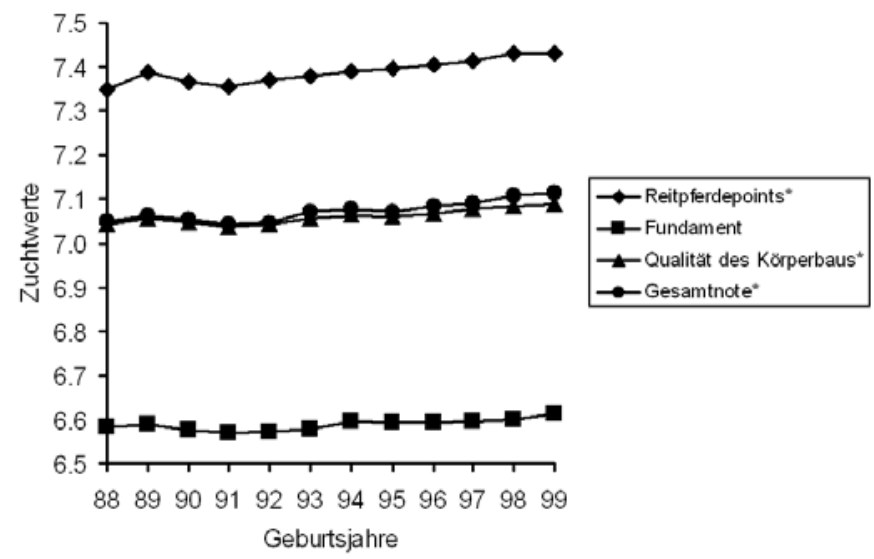

Abb. 1: Genetische Trends der Merkmale Reitpferdepoints, Fundament, Qualität des Körperbaus und Gesamtnote; * signifikanter Anstieg (Genetic trends of the traits horse points, foundation, quality of conformation and total score; * significant increase) 
Mit Ausnahme des Fundamentes sind die genetischen Trends der zusammengefassten Noten, gebildet als Mittelwerte der Zuchtwerte in den einzelnen Geburtsjahren (Abb. 1), im Unterschied zu fast allen anderen Einzelnoten signifikant positiv. Dabei wurden die Geburtsjahre vor 1988 wegen geringer Besetzung nicht berücksichtigt. Die Noten zeigen aufgrund ihrer hohen genetischen Korrelationen einen synchronen Verlauf. Die Geburtsjahrgänge nach der Wende brachten einen leichten Rückschritt. Seitdem verzeichnet die Population einen kontinuierlichen genetischen Fortschritt. Die Abbildungen 2, 3 und 4 dienen der Frage, ob zwischen den Warmblutrassen, den Altersklassen und der Zugehörigkeit zu Verbänden genetische Unterschiede bestehen. Klassen mit geringen Besetzungen wurden in den Abbildungen nicht berücksichtigt. In den genannten Abbildungen ist zu sehen, dass mit Ausnahmen beim Fundament und bei der Gesamtnote signifikante Unterschiede zwischen den Stufen dieser Faktoren in den zusammengefassten Noten gefunden wurden. Die Abbildungen 5 und 6 illustrieren, dass zwischen den Orten, die Stufe 0 kennzeichnet alle nicht angegebenen Orte, und den Monaten der Stutbuchaufnahme, in denen häufig Aufnahmen stattfinden, signifikant unterschiedliche Bedingungen zur Vergabe der zusammengefassten Noten bestanden.

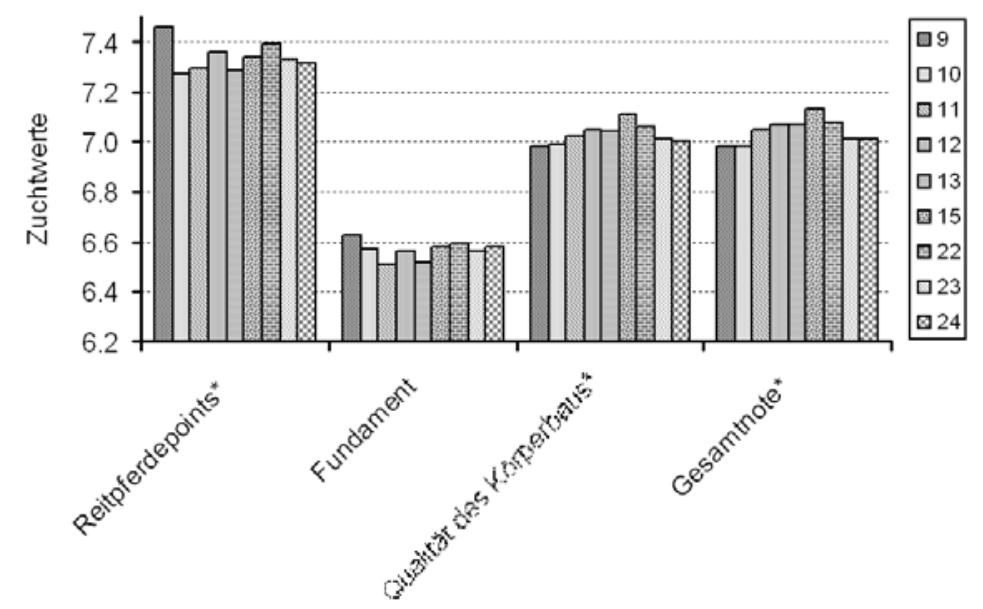

Abb. 2: Mittelwerte von Zuchtwerten innerhalb Rassen; * signifikant different, (Means of breeding values within breeds; * significantly different)

9 Trakehner; 10 Deutsches Reitpferd; 11 Holsteiner; 12 Hannoveraner; 13 Oldenburger; 15 Westfale; 22 Mecklenburger; 23 Brandenburger; 24 Sachsen-Anhaltiner

\section{Diskussion}

Die Noten zur Stutbuchaufnahme sind subjektive Bewertungen des Körperbaus, des Typs und der Bewegung von Stuten. Obwohl für die einzelnen Noten eine Beschreibung von „sehr schlecht“ für Note 1 bis „ausgezeichnet“ für Note 10 gegeben ist, bleibt es dem einzelnen Richter vorbehalten, was einem „ziemlich gut“ für Note 7 bzw. einem „gut“ für Note 8 zuzuordnen ist. Da jedoch innerhalb von Zuchtverbänden auf ein möglichst gleiches Team an Richtern orientiert wird, erscheinen direkte Vergleiche von Noten innerhalb von Zuchtpopulationen möglich. Sie sind jedoch darüber hinaus kaum wertvoll. GRUNDLER und PIRCHNER (1991) stellten in ihrer Studie zur Wiederholbarkeit der Beurteilung von Exterieurmerkmalen fest, dass Richter sich signifikant in ihren Bewertungen unterscheiden und somit als Einflussgröße zu be- 
rücksichtigen sind. Es bestehe jedoch zwischen den Richtern eine gute Übereinstimmung in der Rangierung der Pferde.

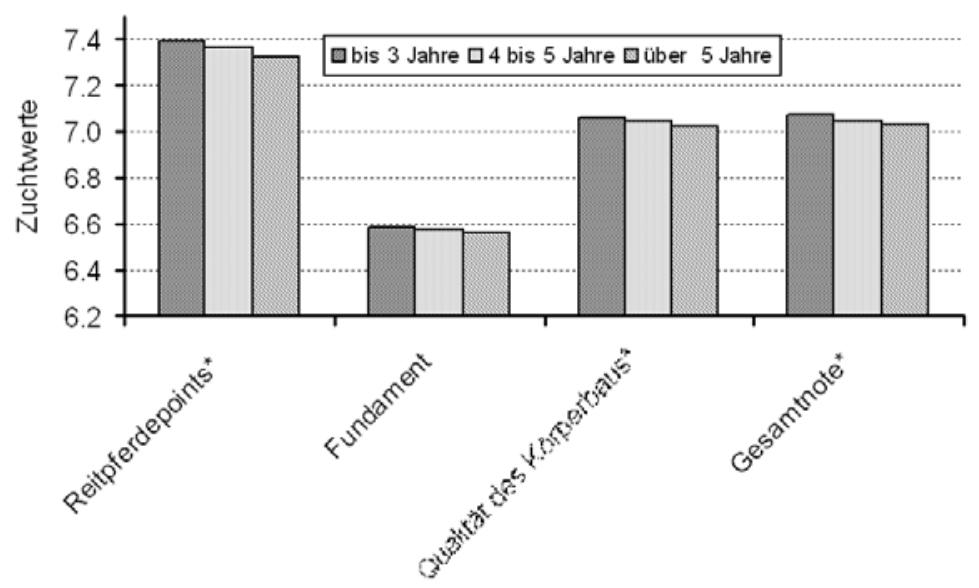

Abb. 3: Mittelwerte von Zuchtwerten innerhalb Altersklassen; * signifikant different (Means of breeding values within classes of age; * significantly different)

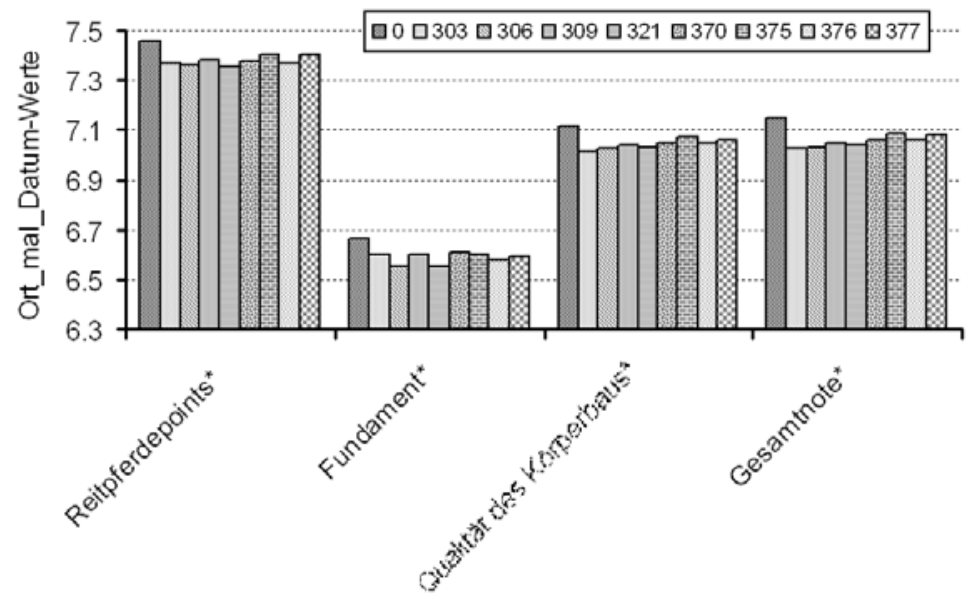

Abb. 4: Mittelwerte von Ort_mal_Datum-Werten innerhalb Orte; * signifikant different (Means of place by date values within places; * significantly different)

303...Sommerstorf; 306...Ganschow; 309...Redefin; $\quad 321 .$. Parkentin; $\quad 370 .$. Gadebusch; 375...Trinwillershagen; 376...Alt Kamp; 377...Altenhagen

Ein Vergleich der genetischen Parameter des Mecklenburger Warmbluts mit anderen Populationen ist am besten mit denen des Hannoveraner Warmbluts möglich, da in beiden Verbänden dieselben Merkmale bonitiert werden. WEYMANN und GLODEK (1991) und CHRISTMANN (1997) schätzten für die Hannoveraner denen der Mecklenburger sehr ähnliche Heritabilitätskoeffizienten. Sie unterscheiden sich im Mittel auch nur geringfügig von denen der Hengste (SCHADE et al., 1996). Der größte Unterschied zeigt sich in der Sattellage, wo in den genannten Arbeiten mit 0,35 bzw. 0,37 ein mehr als dreifach höherer Wert ermittelt wurde. Ebenfalls höher sind die Schätzwerte der Heritabilität für den Rahmen und für die Hintergliedmaßen, deren Wert im Mecklenburger Warmblut mit $8 \%$ extrem niedrig ausfällt. $\mathrm{Zu}$ vermuten ist, dass Fluktuationen in der personellen Besetzung und temporäre Änderungen in den Auffas- 
sungen zum Merkmal wesentliche Ursachen sein können. Bis 1995 hat durch die Berufung hauptsächlich örtlicher Richter ein stärkerer Wechsel in der Besetzung der Kommission stattgefunden. Damit verbunden waren Änderungen in der Schwerpunktsetzung bei der Beurteilung. So sind Wechsel in der Betonung von Widerist und Schulter zur Bewertung der Sattellage bekannt. Treffen diese Vermutungen zu, so sollte sich die Genetik der Merkmale Sattellage, Rahmen und Hintergliedmaßen im Zeitraum bis 1995 von derjenigen nach 1995 unterscheiden. Als Maß bietet sich der genetische Korrelationskoeffizient zwischen „bis 1995“ und „nach 1995“ desselben Merkmals an, der bei gleicher Genetik nur geringfügig kleiner als 1 erwartet wird. Die gefundenen Werte von 0,87und 0,84 bezüglich der Merkmale Sattellage und Rahmen verweisen auf lediglich moderate Unterschiede zwischen beiden Zeitspannen, wobei Änderungen innerhalb der Zeiträume hier nicht erfasst wurden. Die genetische Korrelation von 0,4 des Merkmals Hintergliedmaßen lässt jedoch auf massive Änderungen zwischen den untersuchten Zeitintervallen schließen und kann als Hauptursache der extrem geringen Erblichkeit angesehen werden.

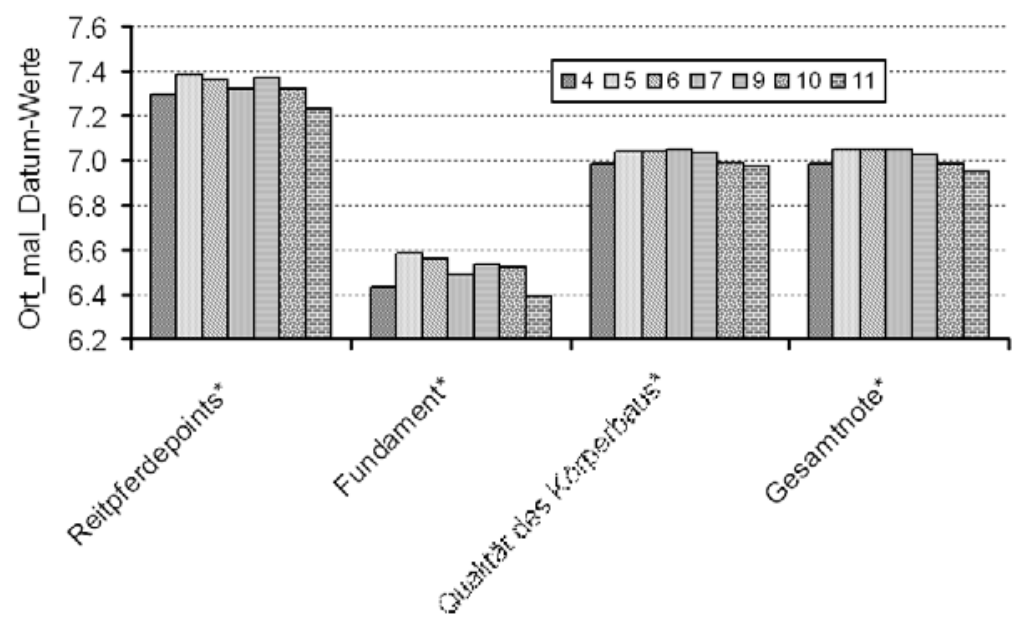

Abb. 5: Mittelwerte von Ort_mal_Datum-Werten innerhalb Monate; * signifikant different (Means of place by date values within months; * significantly different)

Diese Unterschiede wirken sich offensichtlich auf die Gesamtbeurteilung der Stuten nur wenig aus, denn die Heritabilität der Gesamtnote ist im Mecklenburger Warmblut höher als die der Hannoveraner. Vergleiche mit Holsteinern und Brandenburgern sind aufgrund unterschiedlicher Merkmale auf wenige Noten beschränkt. In diesen Noten unterscheiden sich die Ergebnisse von KÜHL et al. (1994) sowie BÖSCH et al. (2000) für die Holsteiner Zucht nur geringfügig von den Werten der Mecklenburger.

Alle Schätzwerte genetischer Korrelationskoeffizienten (Tabellen 3, 4 und 5) sind positiv, im Minimum mit 0,11 zwischen Vordergliedmaßen und Kopf und im Maximum 0,99 zwischen Rasse- und Geschlechtstyp und Reitpferdepoints. Dies schließt, sofern nicht gegen eines oder mehrere Merkmale gezüchtet werden soll, Kontraselektionen im Merkmalsspektrum der Stutbuchaufnahme aus. Die Zuchtwerte der Merkmale Rasse- und Geschlechtstyp und Gesamteindruck stimmen zu mehr als $90 \%$ in ihrer Varianz mit der Qualität des Körperbaus und der Gesamtnote überein. Die besonders hohe Einflussnahme der Note Rasse- und Geschlechtstyp auf den Körperbau stellten auch KÜHL et al. (1994) fest. Im Vergleich zum Hannoveraner Warmblut treten nur 
selten wesentliche Unterschiede zwischen den genetischen Korrelationskoeffizienten auf. Auffällig ist das Merkmal Hintergliedmaßen, bei dem zu den Noten der anderen Merkmale des Körperbaus im Mecklenburger Warmblut deutlich stärkere genetische Beziehungen gefunden wurden. Die phänotypischen Korrelationskoeffizienten sind, wie von KÜHL et al.(1994) ebenfalls berichtet, immer positiv und fast ausschließlich kleiner als ihre genetischen Pendants.

Die Abbildung 1 zeigt, dass sich die Mecklenburger Warmblutpopulation in der Veranlagung der Merkmale der Stutbuchaufnahme positiv entwickelt. Dabei ist hervorzuheben, dass die größten Progressionen nicht in einigen partiellen Eigenschaften zu verzeichnen sind, sondern in der Gesamtheit der äußeren Erscheinung, belegt durch die Gesamtnote, auftritt, was als Indiz einer harmonischen Entwicklung gewertet werden kann. Der Fortschritt in der Gesamtnote wird, und das ist anhand der genetischen Korrelationen zu erwarten, vor allem durch die Merkmale Qualität des Körperbaus und Gesamteindruck getragen, deren Trend neben dem der Gesamtnote statistisch gesichert werden konnte. Positive Trends, hier nicht dargestellt, sind ebenfalls in den Merkmalen Rasse- und Geschlechtstyp, Kopf, Schritt und Elastizität erkennbar. Im Merkmal Rahmen zeichnet sich ein leicht negativer Trend ab.

Die Abbildung 2 belegt, dass zwischen den Warmblutrassen genetische Differenzen erhalten blieben. Signifikante Einflüsse der Rasse findet man auch in den Merkmalen Kopf, Rasse- und Geschlechtstyp, Elastizität, Schritt und Gesamteindruck. Eine besondere Position nimmt dabei das Westfälische Warmblut ein.

Stuten werden vor allen dann erst in höherem Alter zur Beurteilung vorgestellt, wenn die dreijährige Stute in der Entwicklung noch nicht ausgereift erscheint. Die Abbildung 3 belegt, dass dies auch häufig auf eine geringere Veranlagung zurückgeführt werden kann. Für diesen Faktor wurden neben den abgebildeten Merkmalen auch signifikante Einflüsse bei den Merkmalen Sattellage, Elastizität und Gesamteindruck gefunden.

Die Analyse der Zufallsvariablen Ort mal Datum der Stutbuchaufnahme ergibt Hinweise auf Umwelteinflüsse, die sich auf die Wahl von Ort und Zeit der Stutenbewertung zurückführen lassen. Tabelle 2 belegt, dass bei den zusammengefassten Noten 20 $\%$ bzw. 22 \% der phänotypischen Varianz, das sind jeweils etwa 2/3 der genetischen Varianz, durch Ort und Zeit bestimmt werden. Das macht eine Berücksichtigung dieser Faktoren im Modell unabdingbar, weist aber auch auf Reserven in der Gestaltung einheitlicherer Prüfbedingungen hin. Die separate Betrachtung des Ortes der Stutbuchaufnahme (Abb. 4) stellt heraus, dass Bewertungen ohne Kennzeichnungen des Ortes deutlich höher ausfallen. Der Einfluss des Ortes ist neben den in der Abbildung 4 aufgeführten Noten auch bei allen anderen Bewertungsmerkmalen signifikant. Aus Abbildung 5 ist ersichtlich, dass in den mittleren Monaten die Bewertungen ausgeglichener sind. In den anderen Monaten sind die Wertungen signifikant geringer, auch bei den nicht dargestellten Merkmalen.

\section{4.} Schlussfolgerungen

Die ermittelten genetischen Parameter und die gefundenen genetischen Trends bestätigen, dass der Mecklenburger über die Stutbuchaufnahme erfolgreich auf Merkmale der äußeren Erscheinung und der Bewegung gezüchtet werden kann und wurde. Es bestehen jedoch Reserven, wie bei anderen Populationen ebenfalls, im Ausnutzen der Breite der Notenskala und in der Gestaltung einheitlicherer Bedingungen zur Beurteilung der 
Stuten. Ein Übergang zur aufwendigeren linearen Beschreibung, die verbandsübergreifend praktiziert werden müsste, lässt Vorteile im Fortschritt und im Nutzen der Ergebnisse durch den Züchter erwarten.

Die durchweg positiven und teilweise sehr hohen Korrelationen sollten Anlass sein, nur wenige Merkmale, die untereinander schwach korrelieren, im Zuchtziel zu berücksichtigen. Die Erfassung zusätzlicher Merkmale, wie bisher, sollte jedoch beibehalten und der Kontrolle einer harmonischen Entwicklung der Population dienen. Den Ursachen ungenügenden Fortschritts und niedriger Heritabilitäten einzelner Merkmale ist nachzugehen.

\section{Danksagung}

Unser Dank richtet sich an den Verband der Pferdezüchter Mecklenburg - Vorpommern e.V. für das Überlassen der Daten und an das VIT Verden, besonders an Frau Dr. Jaitner, für die Aufbereitung und Übergabe der Dateien.

BRUNS, E.:

\section{Literatur}

Zuchtplanung auf populationsgenetischer Grundlage. Löwe, H. und Hartwig, W., Pferdezucht, S. 308328 Ulmer Verlag (1988)

BÖSCH, M.; REINECKE, S.; RÖHE, R.; KALM, E.:

Genetische Analysen von Merkmalen der Reitpferdezucht - Varianzkomponentenschätzung für Merkmale der Fohlenbeurteilung, Stutbuchaufnahme (Exterieurbeurteilung) und Zuchtstutenprüfung. Züchtungskunde, 72 (2000), 161-171

GROENEVELD, E.; KOVAC, M.:

A Generalised Computing Procedure for Setting up and Solving Mixed Linear Models. J. Dairy Sci., Champaign, III. 73, (1990), 513-531

GROENEVELD, E.:

VCE4 User's Guide and Reference Manual Version 1.1

GRUNDLER, C.; PIRCHNER, F.:

Wiederholbarkeit der Beurteilung von Exterieurmerkmalen und Reiteigenschaften. Züchtungskunde, 63, (1991), 273-281

KÜHL, K.; PREISINGER, R.; KALM, E.:

Analyse von Leistungsprüfung und Entwicklung eines Gesamtzuchtwertes für die Reitpferdezucht. Züchtungskunde, 66 (1994), 1-13

SCHADE, W.; BRUNS, E.; GLODEK, P.:

Züchterische Nutzung der künstlichen Besamung in der Hannoverschen Reitpferdezucht. Arch. Tierz., Dummerstorf 39 (1996), 347-359

SCHWARK, H.J.; PETZOLD, P.; NÖRENBERG, I.:

Untersuchungen zur Auswahl von Selektionskriterien bei der Weiterentwicklung der Reitpferdezucht der DDR. Arch. Tierz., Berlin 31 (1988), 279-289

Eingegangen: 28.01.2004

Anschriften der Verfasser

Dr. GERHARD DIETL

Forschungsinstitut für die Biologie landwirtschaftlicher

Nutztiere, FB Genetik und Biometrie

Wilhelm-Stahl-Allee 2

D-18196 Dummerstorf

E-Mail: dietl@fbn-dummerstorf.de

Dr. SIEGFRIED HOFFMANN

Landesforschungsanstalt Mecklenburg-Vorpommern,

Landestierzucht und Tierzuchtanerkennung

Dorfplatz 1

D-18276 Gülzow

E-Mail: s.hoffmann@lfa.mvnet.de
Akzeptiert: 26.02.2004

SATU ALBRECHT

Gockelgasse 14a

D-18069 Sievershagen

E-Mail: sa_al@gmx.de 